\title{
Domain Drivers in the Modularization of FLOSS Systems
}

\author{
Andrea Capiluppi \\ Centre of Research on Open Source Software, \\ University of Lincoln, Brayford Campus, Lincoln, \\ LN5 7TS, United Kingdom \\ acapiluppi@hemswell.lincoln.ac.uk
}

\begin{abstract}
The classification of software systems into types has been achieved in the past by observing both their specifications and behavioral patterns: the SPE classification, for instance, and its further supplements and refinements, has identified the S-type (i.e., fully specified), the P-type (i.e., specified but dependent on the context) and the E-type (i.e., addressing evolving problems) among the software systems.

In order to detect types, and establish similarities, among Free/Libre/Open Source Software (FLOSS) systems, this paper considers three modular characteristics (functions, files and folders) and their evolution: how they are evolving with size, if they are constant across systems, and whether recurring evolutionary patterns are observed. Using these various-grained characteristics, a set of models for the evolution of modularization are extracted from evolving systems, and then used to extract similarities and types from a wide sample of FLOSS projects.

This paper provides three contributions: first, it shows that several models are needed to encompass the variety of modularization patterns; second, it provides three types of models (uni-variate, bi-variate and tri-variate) for the evolution of modularization, with significant goodness-of-fit's. Finally, it shows that two of these patterns alone can interpolate the modular characteristics of the vast majority of a random choice of FLOSS projects.
\end{abstract}

\section{Introduction and Related Work}

The classification of software systems into types, if properly conducted, can serve diverse purposes: by classifying and indexing objects or components, for example, developers can ease the search and location of reusable software [8,21]. As a matter of fact, any reuse effort always involves two major steps to achieve its results: first, it must be able to clearly identify reusable components; second, it must put in place a library of reusable entities for their selection in future projects [15[16]. As a necessary condition for these two steps, a reusable component should be uniquely classified and described, its functionalities and application domain clearly identified, and its inward and outwards connections described.

Classification of software systems has also been achieved in the past with the purpose of assigning common features of frequently observed patterns to categories, like the SPE program classification [13]. Based on this, a software system can be classified

C. Boldyreff et al. (Eds.): OSS 2009, IFIP AICT 299, pp. $3-19,2009$.

(c) IFIP International Federation for Information Processing 2009 
as S-type, when "the specification is the complete, sole and definitive determinant of program properties" [12]; a software system is instead classified as P-type when "the result from the execution of the program is correct in a sense provided by the problem statement" [12]. Given the definition, a P-type system can be considered as S-type as long as its underlying problem was stated completely and precisely. Finally, an E-type system has been described as implementing an application in a "real world domain", and its overall value is only in part dependent on the correctness of the expected outcome, other aspects being the interaction with users and other components. More recently, the same SPE classification was adapted to comprise patterns of behavior and evolution [5].

Finally, from an evolutionary perspective, the classification of software systems has proven useful to identify the presence of patterns of evolution. In recent works, either similarity or volatility, (i.e., variety of behaviors, or absence of similarity) has been used to characterize the evolution of both commercial [1] and FLOSS products [2 10 23] and processes [17].

This study builds upon two core concepts of the software engineering knowledge, namely the classification of software systems, as briefly introduced above, and their modularity [19]. The decomposition of software systems into smaller modules, each with large internal cohesion and low coupling with others, is an established framework for software designers and architects. Modules form basic building blocks, and their dimension are typically accomplished avoiding both too large (i.e. under-modularization) or too small (i.e. over-modularization) components [20].

This paper studies the evolution of three modular characteristics (source functions or methods, source files and source folders) with respect to the size of the system in order both to detect patterns of modular evolution, and to identify clusters or types in the modularization of 26 FLOSS systems evolution. Since types and patterns are here expressed in terms of multivariate models, the paper will initially assess whether a single model could instead be fitted for all the systems. Later, these empirical models will be used to interpolate the modular characteristics of two random samples of FLOSS projects.

This paper is articulated as follows: Section 2 will introduce the definitions and the empirical hypothesis that this study is based upon, since it proposes multiple, multivariate models. Section 3 will test the hypothesis by introducing a cross-sectional analysis: all of the observed systems will be put in the same evolutionary pool, and a single model will be sought. Section 4 will at first discuss the problem of multi-collinearity when dealing with multi-variate models. Then it will investigate and display the models which were extracted from the evolutionary history of the 26 FLOSS systems. Section 5 will use these models to evaluate the modular characteristics of a the second sample of FLOSS systems, and to assess which of these models better formalizes what system. Finally, Section 6 will present the conclusions and illustrate potential future works.

\section{Definitions}

From the theoretical standpoint, modularity of FLOSS projects has been extensively analyzed [7 14 18], and advocated as a necessary condition for appropriately leveraging the distributed approach of FLOSS developers. Less frequently it has been evaluated empirically, and mostly on fine-granular elements: for example, the modularization (in 
terms of functions) in FLOSS procedural languages has been compared to the modularization (in terms of methods) in FLOSS object-oriented languages [6]; also, the common coupling among modules of the Linux kernel has been extensively analyzed [25].

The terminology and definitions used in this paper are therefore extracted from similar studies in the past FLOSS literature [3]9 10|22], especially those related to entities with different levels of granularity. The empirical hypothesis that this paper is built upon is presented in Section 2.1.

1. Source function: basic unit of source code; this term is used to refer to procedures, subroutines, but also OO-methods.

2. Source file: any file with at least one source function.

3. Source folder: any folder containing at least one source file [4]. The term module is used here to refer to source code functions, files and folders.

4. Size: the length of the whole system, of a folder, a file or a function, which can be evaluated at different levels of granularity, for example: number of folders, of files, lines of code (LOC) and lines of source code (SLOC) which excludes blank lines and embedded comments.

5. Application domain: The application domains of the sample has also been studied. These domains are those used within a well known FLOSS repository (the SourceForge site) to effectively cluster the projects. Table 1 summarizes the domains and the relative keys used throughout the paper.

Table 1. Application domains as used in the SourceForge repository

\begin{tabular}{|c|c|}
\hline Application Domain & Key \\
\hline \hline Communications & $\mathrm{A}$ \\
\hline Database & $\mathrm{B}$ \\
\hline Desktop Environment & $\mathrm{C}$ \\
\hline Education & $\mathrm{D}$ \\
\hline Formats and Protocols & $\mathrm{E}$ \\
\hline Games/Entertainment & $\mathrm{F}$ \\
\hline Internet & $\mathrm{G}$ \\
\hline Multimedia & $\mathrm{H}$ \\
\hline Office/Business & $\mathrm{I}$ \\
\hline Other/Nonlisted Topic & $\mathrm{J}$ \\
\hline Printing & $\mathrm{K}$ \\
\hline Scientific/Engineering & $\mathrm{L}$ \\
\hline Security & $\mathrm{M}$ \\
\hline Software Development & $\mathrm{N}$ \\
\hline System & $\mathrm{O}$ \\
\hline Terminals & $\mathrm{P}$ \\
\hline Text Editor & $\mathrm{Q}$ \\
\hline
\end{tabular}

6. Programming language: this paper will differentiate, for each project, between a procedural $(\mathrm{P})$ and object-oriented $(\mathrm{OO})$ paradigm. This distinction will be made based on a prevalence (i.e. more than $80 \%$ ) of one specific programming language 
(and paradigm) over others (4th column of Table 2). In cases where multiple programming languages (and paradigms) are present with similar shares, an appropriate notation is used. As an example, the project with Id 5 is composed of $\mathrm{C}$ source files (40\%) and the rest of the Dylan (also procedural) programming language.

Table 2. Summary of Programming paradigms and Application Domains

\begin{tabular}{|c||c|c|c|c|}
\hline $\mathrm{Id}$ & Project & Releases & Language & Domain \\
\hline \hline 1 & abiword & 82 & $\mathrm{OO} / \mathrm{P}$ & $\mathrm{Q}$ \\
\hline 2 & arla & 68 & $\mathrm{P}$ & $\mathrm{G}$ \\
\hline 3 & gaim & 98 & $\mathrm{P}$ & $\mathrm{A}$ \\
\hline 4 & ganymede & 42 & $\mathrm{OO}$ & $\mathrm{G}$ \\
\hline 5 & gdylan & 17 & $\mathrm{PP}$ & $\mathrm{M}$ \\
\hline 6 & ghemical & 21 & $\mathrm{OO}$ & $\mathrm{L}$ \\
\hline 7 & gimp_print & 117 & $\mathrm{OO}$ & $\mathrm{K}$ \\
\hline 8 & gimp_stable & 34 & $\mathrm{OO}$ & $\mathrm{H}$ \\
\hline 9 & gimp_dev & 96 & $\mathrm{OO}$ & $\mathrm{H}$ \\
\hline 10 & gist & 19 & $\mathrm{OO}$ & $\mathrm{G}$ \\
\hline 11 & grace & 36 & $\mathrm{P}$ & $\mathrm{L}$ \\
\hline 12 & htdig & 17 & $\mathrm{P}$ & $\mathrm{G}$ \\
\hline 13 & ksi & 14 & $\mathrm{P}$ & $\mathrm{M}$ \\
\hline 14 & lcrzo & 56 & $\mathrm{P}$ & $\mathrm{G}$ \\
\hline 15 & motion & 81 & $\mathrm{P}$ & $\mathrm{H}$ \\
\hline 16 & mplayer & 77 & $\mathrm{P}$ & $\mathrm{H}$ \\
\hline 17 & mrtg & 77 & $\mathrm{P}$ & $\mathrm{G}$ \\
\hline 18 & mutt & 91 & $\mathrm{P}$ & $\mathrm{A}$ \\
\hline 19 & netwib & 35 & $\mathrm{P}$ & $\mathrm{G}$ \\
\hline 20 & rrdtool & 35 & $\mathrm{PP}$ & $\mathrm{O}$ \\
\hline 21 & siagoffice & 46 & $\mathrm{P}$ & $\mathrm{I}$ \\
\hline 22 & vovida & 14 & $\mathrm{OO} / \mathrm{P}$ & $\mathrm{A}$ \\
\hline 23 & wine_stable & 20 & $\mathrm{P}$ & $\mathrm{O}$ \\
\hline 24 & wine_unstable & 90 & $\mathrm{P}$ & $\mathrm{O}$ \\
\hline 25 & xfce & 67 & $\mathrm{P}$ & $\mathrm{C}$ \\
\hline 26 & xmms & 29 & $\mathrm{P}$ & $\mathrm{H}$ \\
\hline
\end{tabular}

\subsection{Working Hypothesis}

Previous studies have been conducted to inform about the presence of correlation between

a the size of FLOSS systems and

b time of development (in days, weeks or months)

showing very high goodness-of-fit [2[10]. It is argued here that these models present severe pitfalls: the set of resulting models not only lacks information on how modularization is achieved, but also establishes a relation between an internal attribute (i.e. size, in SLOCs) and an external measurement (i.e. time, in days, weeks or months). This paper will explore evolutionary models comprising an internal dependent variable (size), 
and internal independent variables (number of source folders, files and functions). This will in turn remove any modeling distortions resulting from long periods of inactivity or peaks of activity (as seen in FLOSS systems in proximity of major releases [9]).

The working hypothesis underlying this study states that a single modularization model cannot encompass the variety of FLOSS observed evolutionary patterns. In terms of null hypothesis (H0), the model [m0]

$$
\text { size }=a * \text { folders }+b * \text { files }+c * \text { functions }+d[\mathrm{~m} 0]
$$

will produce an adequate goodness-of-fit for all the selected FLOSS projects. The empirical evaluation of this will be achieved analyzing the level of significance of the four parameters (a, b, c and d), i.e. evaluating their t-value's and p-value's.

The alternative hypothesis, H1, requires that several models are necessary to fit the modularization patterns of FLOSS projects. As a summary, Table 3 displays the null and the alternative hypotheses, their description, and how they will be tested.

Table 3. Summary of the research hypotheses

\begin{tabular}{|l|l|l|}
\hline Type & Description & Measures \\
\hline \hline H0 & $\begin{array}{l}\text { Single model [m0] for all FLOSS sys- } \\
\text { tems }\end{array}$ & $\begin{array}{l}\text { t-value's of a,b,c,d large; p-value's of } \\
\text { a,b,c,d } \leq 0.2\end{array}$ \\
\hline H1 & Multiple models needed & $\begin{array}{l}\text { t-value's of a,b,c,d small; p- value's of } \\
\text { a,b,c,d }>0.2\end{array}$ \\
\hline
\end{tabular}

\section{Cross-Sectional Analysis}

A cross-sectional study design [11] is used in this section for validating and testing the research hypothesis. This type of statistical test is ideal for the proposed hypothesis, since it builds a very basic form of understanding of the data. In this case, it helps in detecting whether a generic, overall model can be established between the dependent variable (size, in LOCs) and the modular characteristics (source folders, files and functions). In a cross-sectional analysis, either the entire population or a subset is selected, in a single snapshot (i.e. no longitudinal analysis is performed): in the case depicted by this paper, the overall population of the 26 FLOSS projects was put in the same statistical pool to detect a unique relationship.

\subsection{Design of the Experiment}

The purpose of the investigation is to assess the significance of the modularization model [m0]: each of the parameters (a,b,c and d) will be extracted from the data of all the systems, together with its level of confidence, in terms of t-value and p-value. This was repeated several times, in a stratified approach: the steps below summarize the design and implementation of the statistical analysis.

1. At first, the systems in Table 2 were ordered by number of available releases, and a lower limit was set as a minimum to conduct the study: a minimum threshold of 29 releases was selected as the first cross-section (first row of Table 4), as it appeared to be large enough to collect statistical data. 
2. All the systems with exactly or more than 29 releases (therefore excluding projects with ID's 5, 6, 10, 12, 13, 22, 23 from Table 2) were listed, and their latest 29 releases, with their data on source folders, files and functions, comprising 551 data points, formed the first population, for which the first multi-variate regression model was calculated. The coefficients of the model, as well as the determination coefficient $\left(R^{2}\right)$ were evaluated (4th, 5th, 6th and 3rd columns of Table 4).

3. As a second step, the number of releases closest and larger than 29 was selected as the next threshold (i.e., 34). As done previously, all the systems with exactly or more than this threshold of releases were considered (hence excluding project with ID 8 from Table 2), forming a pool of 612 data points. As before the coefficients of the multi-variate regression were evaluated, together with the $R^{2}$ factor.

4. The same approach was applied, recursively, for the ordered number of releases as cross-sections. A decreasing number of projects participated to the various studies, and different pools of data points (2nd column of Table 4) were considered, as per definition of cross-sectional design.

5. At the end of all the iterations, the mean and the variance of the coefficients were evaluated, and later used to evaluate the t-value and the p-value of each attribute.

\subsection{Results of the Cross-Sectional Study}

The results of the set of steps as briefly summarized above analysis are displayed in the last four rows of Table 4: the t-value's and p-value's are reported for each of the independent variables (folders, files and functions) and the intercept ("Const"). The only confidence achievable is on the two regressed parameters "Funct" and "Const", while the parameters of "Folder" and "File" have a low t-value and a high p-value.

As per the definitions given in Table 3 , it is possible to reject the null hypothesis H0: from the sample of 26 FLOSS projects, it's not possible to extract one single modularization model. The variety of observed behaviors (in terms of modularization) of the selected systems requires a larger set of models: in the next sections, each FLOSS project will be analyzed to discover one or more patterns of evolution of modularization, and the problem of multi-collinearity will be discussed.

\section{Evolutionary Models}

The previous Section 3 showed that a single multi-variate correlation, comprising the characteristics of source folders, files and functions, can not represent, on its own, the modularization patterns of the considered FLOSS projects.

One solution to address this issue would be to refine the model to make it inclusive of all the variations of the ob- served behaviors. Another solution is instead to investigate each project in order to detect the presence of one or more modularization models. This second option is more reasonable, also from previous empirical evidence on FLOSS projects [2]10 24], which already shows diverse patterns of evolution.

In the next subsection, the problem of multi-collinearity [1124] is investigated in order to detect (if any) the principal modular characteristics of each project's evolution, and to discard the non-relevant ones. 
Table 4. Cross-sectional design study - results

\begin{tabular}{|c|c|c|c|c|c|c|}
\hline Cross-Sections & Data points & $R^{2}$ & Dir & File & Funct & Const \\
\hline 29 & 551 & 0.957 & 422.08 & 77.76 & 32.39 & 26519.83 \\
\hline 34 & 612 & 0.957 & 330.94 & 70.5 & 33.48 & 28873.35 \\
\hline 35 & 595 & 0.958 & 627.72 & 65.36 & 31.31 & 25449.24 \\
\hline 35 & 560 & 0.957 & 625.78 & 66.11 & 31.33 & 24121.65 \\
\hline 36 & 540 & 0.958 & 568.18 & 60.75 & 32.28 & 23600.43 \\
\hline 42 & 588 & 0.959 & 477.4 & 55.43 & 33.51 & 21133.54 \\
\hline 46 & 598 & 0.960 & 364.32 & 48.24 & 35.23 & 18447.44 \\
\hline 56 & 672 & 0.965 & 13.9 & 32.86 & 39.18 & 27244.26 \\
\hline 67 & 737 & 0.965 & -47.38 & 26.79 & 40.12 & 27348.65 \\
\hline 68 & 680 & 0.988 & -1239.44 & 15.42 & 50.22 & 28085.51 \\
\hline 77 & 693 & 0.986 & \begin{tabular}{|l|}
-930.71 \\
\end{tabular} & 9.45 & 48.53 & 24898.57 \\
\hline 77 & 616 & 0.985 & -871.73 & 9.23 & 48.01 & 26336.04 \\
\hline 81 & 567 & 0.989 & -804.14 & -4.67 & 48.89 & 20642.72 \\
\hline 82 & 492 & 0.987 & -749.78 & -5.58 & 48.16 & 27414.36 \\
\hline 90 & 450 & 0.990 & 107.53 & 91.9 & 35.36 & 18794.66 \\
\hline 91 & 364 & 0.985 & -17.44 & 24.75 & 46.41 & 13399.28 \\
\hline 96 & 288 & 0.983 & 32.28 & 21.87 & 47.01 & 7075.48 \\
\hline 98 & 196 & 0.893 & 1992.91 & -308.17 & 50.08 & 30839.27 \\
\hline 117 & 117 & 0.267 & -667.08 & -24.08 & 32.24 & 56036.34 \\
\hline \multicolumn{3}{|c|}{ Mean } & 12.39 & 17.57 & 40.2 & 25066.35 \\
\hline \multicolumn{3}{|c|}{ Variance } & 765.83 & 85.06 & 7.59 & 9476.44 \\
\hline \multicolumn{3}{|c|}{ T-value } & 0.016 & 0.207 & 5.297 & 2.645 \\
\hline \multicolumn{3}{|c|}{ P-value (18 d.f.) } & 0.987 & 0.838 & 0.000 & 0.017 \\
\hline
\end{tabular}

\subsection{Addressing Multi-collinearity}

In its definition, multi-collinearity is the presence of a significant linear relationship (reflected, for instance, by a large value of $R^{2}$ ) between two or more independent (or explanatory) variables. The presence of multi-collinearity poses serious problems when defining the relevance of a variable into the regressed model: for instance, one could overestimate the weight of a variable even if it was perfectly correlated (i.e. superfluous) to another one [24].

In order to refine the single model as expressed above, each system was therefore studied on its own, and the characteristics of source folders, files and functions were taken as independent variables and the size in LOCs as dependent variable. This was repeated in all the releases: finally, the correlation among the independent variables was studied. An acceptable multi-variate regression must have low correlations among the independent variables, and should have high correlation between each independent variable (folder, file, function) and the dependent one (size).

Generating Models from Evolutionary Data. For only 4 projects (ganymede, gimpprint, gist, lcrzo) the three modular characteristics are relevant in the evolutionary behavior (i.e., the $R^{2}$ between each pair of attributes are all $<0.9$ ), thus generating a 


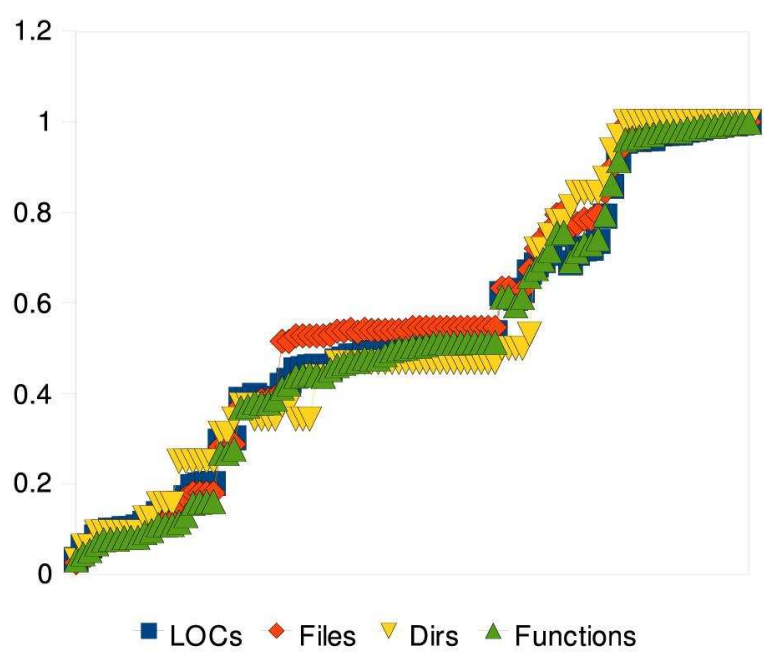

Fig. 1. Evolution trends in the pattern size $=\{$ folders, files, functions $\}$ (Gaim system)

Table 5. Summary - Evolutionary patterns

\begin{tabular}{|l||cc|}
\hline Pattern & Type of pattern & $R^{2} \geq 0.9$ \\
\hline \hline $\mathrm{p} 1$ & size $=f(\{$ folder, file, funct $\})$ & 17 \\
\hline $\mathrm{p} 2$ & size $=f($ folder, file $)$ & 12 \\
\hline $\mathrm{p} 3$ & size $=f($ folder, funct $)$ & 7 \\
\hline $\mathrm{p} 4$ & size $=f($ file, funct $)$ & 2 \\
\hline $\mathrm{p} 5$ & size $=f($ folder, file, funct $)$ & 2 \\
\hline
\end{tabular}

size $=f($ folders, files, functions $)$ pattern. In all the remaining projects, one (or more) multi-collinearity problems were detected, since at least two of the attributes were found to be highly correlated. Therefore, in these systems other patterns were sought: whenever a high correlation was found between two variables, their relevance was questioned compared to the other variables. In cases of evident multicollinearity, one of these variables was dropped.

As an example, the system abiword (Id 1) shows a high correlation between number of folders and number of functions; and between number of functions and number of files: therefore, the variable number of functions was excluded from the model. The model explaining the modularization of the abiword system was chosen to be:

$$
\text { size }=a *(\text { folders })+b *(\text { files })+d[\mathrm{~m} 1]
$$

and coded as the size $=f($ folders, files $)$ pattern in the 5 th column of Table 7 (with key $\mathrm{m} 1)$.

For other projects (gaim, Id 3), no correlation was found to be significant. It was chosen to have a richer set of models in this case: the underlying pattern comprises three models (represented as $\mathrm{m} 3, \mathrm{~m} 4$ and $\mathrm{m} 5$ in Table 7) which jointly represent the pattern: 


$$
\begin{gathered}
\text { size }=a *(n r \text { folders })+d[\mathrm{~m} 3] \\
\text { size }=b *(n r \text { files })+d[\mathrm{~m} 4] \\
\text { size }=c *(n r \text { functions })+d[\mathrm{~m} 5]
\end{gathered}
$$

This complex behavior was coded instead as the

$$
\text { size }=f(\{\text { folders }, \text { files }, \text { functions }\})
$$

pattern in Table 7, meaning that the "size"variable can be explained by any of the "number of files", "number of folders"or "number of functions" alone. We claim that this pattern achieves the best modularization for FLOSS system: all the dependent variables have the same evolutionary trend, and each can explain alone the growth of size. This behavior is visually depicted (when normalized) in Figure 1 for the Gaim system.

Considering the variations of the 26 patterns, a maximum of 45 different models were found, whose attributes ( $a, b, c$ and d) have been evaluated and fully expanded in Table 7. Each of these models was interpolated with evolutionary data in terms of goodness-of-fit. In 6 cases it was found that the corresponding model had a significance lower than 0.9 in terms of $R^{2}$ : this reduced the overall number of models to 39 .

In terms of affected patterns, the summary in Table 5 shows the relevance of each pattern as observed in the whole pool, and considering only those cases where the regression fit was larger than 0.9 and 0.7 . As observed above, the proposed pattern containing the three explaining variables (p5, in the form of

$$
\text { size }=f(\text { folders }, \text { files }, \text { functions })) \text {, }
$$

was empirically observed only in 2 out of 40 statistically relevant patterns (i.e., with $\left.R^{2} \leq 0.9\right)$. Most of the other patterns represent an interaction of at most two variables (21 out of 39) or a single variable (17 cases out of 39). Also, the union of the patterns p1 and p2 alone is responsible for some $3 / 4$ of the extracted models.

\section{Testing the Models}

The relations obtained in the previous Section 4 formed a pool of models, each characterizing the evolution of the size (in LOCs) as a function of the modular characteristics (source folders, files and functions) of a specific project. The goodness of fit of most of these models was demonstrated to be statistically significant via the indicator $R^{2}$.

\subsection{Design of the Experiment}

In this subsection, the models defined above (from $\mathrm{m} 0$ to $\mathrm{m} 40$ ) are used to test whether a generic FLOSS project shows a modularization which can be interpolated by any of the models above.

In order to do so, a random sample of 50 FLOSS projects was extracted from both the Debian and the SourceForge repositories, resulting in 100 projects. Debian contains a popular FLOSS forge which is the basis of the successful Debian distribution; SourceForge hosts more than 200,000 FLOSS projects and is recognized as the most common 
FLOSS portal. The two samples were extracted from similar-sized pools, i.e. the "stable" subset of hosted projects, as per the development status that each FLOSS project can select as its own. For each project in the samples, the number of folders, files and functions were evaluated together with the value of size in SLOCs.

Table 8 shows the summaries of the attributes of these samples: information on the "programming languages" and "application domains" was also collected: depending on the programming language, OO projects was selectively interpolated with OO models, and similarly for procedural projects. For some of the projects, information on source functions could not retrieved, since their languages (like Python, PHP or the such) were not supported by the available tools. These projects were discarded from the model testing.

The modular characteristics of each project were used within a model obtaining an estimated size as the dependent variable: this value was later compared with the real value of size (in SLOCs). The error made by the estimation was evaluated as follows

$$
\text { error }=a b s\left[\frac{\text { Size }_{r e}-\text { Size }_{e s t}}{\text { Size }_{r e}}\right]
$$

where Size $_{r e}$ represents the real size value, and Size $e_{\text {est }}$ the estimate given by the model. The p25 (the probability that the estimate diverges for less than $25 \%$ from the real value) is recorded in Table 6 . The summary differentiates among the application domains of Table 1 and the patterns (p1 to p5) of Table 5: within the projects with application domain "A" (Communication), the first row summarizes that:

- 4 models from Table 7 and pattern p1, i.e. f(folder, file, function), estimate the achieved size of a subset of projects (sharing the same programming paradigm of these models $-\mathrm{OO}$ vs $\mathrm{P}$ ) with an error $<25 \%$;

Table 6. Predictability of patters at $p<0.25$

\begin{tabular}{|c|c|c|c|c|c|}
\hline Pattern & $\mathrm{p} 1$ & $\mathrm{p} 2$ & $\mathrm{p} 3$ & $\mathrm{p} 4$ & $\mathrm{p} 5$ \\
\hline $\mathrm{A}$ & 4 & 1 & 2 & & \\
\hline B & & 2 & 1 & & \\
\hline $\mathrm{C}$ & 5 & 1 & 1 & & \\
\hline \multicolumn{6}{|l|}{ D } \\
\hline $\mathrm{E}$ & & & 1 & & \\
\hline $\mathrm{F}$ & 4 & 3 & 2 & 1 & 1 \\
\hline $\mathrm{G}$ & 11 & 3 & 2 & 1 & 2 \\
\hline $\mathrm{H}$ & 10 & 5 & 3 & 1 & 1 \\
\hline $\bar{I}$ & 9 & 5 & 2 & 1 & 1 \\
\hline $\mathrm{J}$ & & & & & 2 \\
\hline $\mathrm{K}$ & 14 & 4 & 2 & 1 & 2 \\
\hline $\mathrm{L}$ & 16 & 2 & 2 & 1 & \\
\hline $\mathrm{M}$ & & 2 & & & \\
\hline $\mathrm{N}$ & 1 & 1 & 1 & & \\
\hline $\mathrm{O}$ & 3 & 1 & 1 & 1 & \\
\hline Totals & $\overline{77}$ & 30 & 20 & $\overline{77}$ & $\overline{9}$ \\
\hline$\%$ & 53.8 & 20.9 & 13.9 & 4.90 & 6.29 \\
\hline
\end{tabular}


- only one model with the pattern p2 (f(folder, file)) can properly estimate the size in SLOCs of one project with a similar error;

- 2 models with pattern p3 estimate the SLOCs of the two random samples with a comparable error.

\subsection{Results of the Experiment}

The following insights can be drawn from this summary table:

1. As visible in the last two rows, two patterns perform better than others in terms of error made in the estimation of size (in SLOCs). Alone, the patterns p1 and p2 cover 34 of the successful estimates: this means that the models based on one attribute alone (i.e., number of folders, or files, or functions) explain the modularization patterns of the majority of the projects in these samples. The size in SLOCs is therefore predictable using just one attribute, and the ratio SLOCs/attribute represents a constant.

2. A subset of application domains $(\mathrm{B}, \mathrm{D}, \mathrm{E}, \mathrm{J}, \mathrm{M}, \mathrm{N})$ is more difficult to estimate than others. This has two explanations: first, apart from the domain " $\mathrm{M}$ ", the evolutionary sample of Table 2 does not contain projects belonging to this subset. Hence, specific models for these domains were not produced. Second, the two random samples of FLOSS projects also contain few projects (6 overall) belonging to these domains, hence making it difficult to draw conclusions on them.

3. A subset of application domains (F, G, H, I, J, L) is instead attracting several estimates from diverse patterns (although the patterns $\mathrm{p} 1$ and $\mathrm{p} 2$ still prevail). Apart from the " $G$ " (Internet) domain, they all represent front-end user applications (as opposed to back-end system administrators): this result is therefore stating that the projects in these domains have diverse modularization types, ranging from highly modular (as mirrored by the p1 pattern) to the least modular, when the three modular characteristics (folder, file and function) are needed to estimate the size in SLOCs (as mirrored by the pattern p5).

\section{Conclusions}

This paper used publicly available FLOSS data and shared metrics in order to provide a mechanism to classify the evolution of software systems. In past literature, the shapes of evolutionary curves have been qualitatively observed, or univariate models (size-time) have been used to draw similarities among systems. In this study, the relationship between size (as dependent variable) and the modularization characteristics of systems (number of source folders, files and functions - as independent variables) was used to first extract models of evolution, select patterns out of these and then fit these modularization models on a random sample of FLOSS projects.

The study was preceded by a research hypothesis: a unique modularization model can not capture the variety of observed behaviors in FLOSS systems, but a set of modularization models is needed. The model (m0) used as a benchmark was a multi-variate linear correlation, in the form size $=a *$ folders $+b *$ files $+c *$ function $+d$. This hypothesis was tested through a cross-sectional analysis, using the whole set of gathered FLOSS data (summing up to more than 1,300 data points). It was found that the single model could not be considered statistically accurate for each and all the considered systems. 
As a result of the hypothesis testing, the presence of a whole set of modularization models was investigated. For every FLOSS project in the pool, the benchmark model $\mathrm{m} 0$ was analyzed, and the multi-collinearity issue was discussed: for some of the projects, in fact, the multivariate model revealed the presence of collinearity among some of the independent variables. In those cases, a simpler pattern was tested, either with only two independent variables, or just with one. In some of the projects, the multi-collinearity of the variables pointed to a complex pattern, where more than one patterns were used to describe the evolution of modularization. As a result of this step, it was observed a dominance of univariate and bi-variate patterns over the benchmark model, which could be observed as statistically relevant (in terms of $R^{2}$ ) only in 2 out of 39 models.

In order to test these models, and their accuracy in predicting the modularization of FLOSS systems, a random sample of FLOSS projects was extracted from the Debian distribution and the SourceForge portal, and their modular characteristics recorded. The models were used to interpolate the variables, and to predict the size, of the FLOSS projects: models with a specific programming paradigm (OO or procedural) were used to interpolate the FLOSS projects using the same paradigm. This prediction was then compared to the actual size in SLOCs of the project, with an error of $25 \%$.

The first finding of this analysis showed that two patterns stand out in terms of prediction power: $\mathrm{p} 1$ and $\mathrm{p} 2$ could cover up to $3 / 4$ of the successful predictions. The second finding pointed at the uneven distribution of domains in a random sample, showing that specialized topics (Databases, Education) are also more difficult to model. The third finding showed that high-end applications suffer from a high variety of modularization patterns, ranging from very modular models (where each attribute can be considered as constantly growing with the size) to uneven growth of each attribute, resulting in a model where each attribute is needed to interpolate the achieved size.

\section{References}

1. Barry, E.J., Kemerer, C.F., Slaughter, S.A.: On the uniformity of software evolution patterns. In: ICSE 2003: Proceedings of the 25th International Conference on Software Engineering, pp. 106-113. IEEE Computer Society, Washington (2003)

2. Capiluppi, A.: Models for the evolution of os projects. In: ICSM 2003: Proceedings of the International Conference on Software Maintenance, p. 65. IEEE Computer Society, Washington (2003)

3. Capiluppi, A., Boldyreff, C.: Identifying and improving reusability based on coupling patterns. In: Mei, H. (ed.) ICSR 2008. LNCS, vol. 5030, pp. 282-293. Springer, Heidelberg (2008)

4. Capiluppi, A., Morisio, M., Ramil, J.F.: The evolution of source folder structure in actively evolved open source systems. In: METRICS 2004: Proceedings of the Software Metrics, 10th International Symposium, pp. 2-13. IEEE Computer Society, Washington (2004)

5. Cook, S., Harrison, R., Lehman, M.M., Wernick, P.: Evolution in software systems: foundations of the spe classification scheme: Research articles. J. Softw. Maint. Evol. 18(1), 1-35 (2006)

6. Ferrett, L.K., Offutt, J.: An empirical comparison of modularity of procedural and objectoriented software. In: ICECCS 2002: Proceedings of the Eighth International Conference on Engineering of Complex Computer Systems, p. 173. IEEE Computer Society, Washington (2002) 
7. Fitzgerald, B.: A critical look at open source. Computer 37(7), 92-94 (2004)

8. Frakes, W.B., Pole, T.P.: An empirical study of representation methods for reusable software components. IEEE Trans. Softw. Eng. 20(8), 617-630 (1994)

9. German, D.M.: Using software trails to reconstruct the evolution of software: Research articles. J. Softw. Maint. Evol. 16(6), 367-384 (2004)

10. Herraiz, I., Gonzalez-Barahona, J.M., Robles, G.: Towards a theoretical model for software growth. In: MSR 2007: Proceedings of the Fourth International Workshop on Mining Software Repositories, p. 21. IEEE Computer Society, Washington (2007)

11. Lauridsen, J., Mur, J.: Multicollinearity in cross-sectional regressions. Journal of Geographical Systems 8(4), 317-333 (2006), http: / / ideas.repec.org/a/kap/jgeosy/ v8y2006i4p317-333.html

12. Lehman, M.M.: Uncertainty in computer application and its control through the engineering of software. Journal of Software Maintenance 1(1), 3-27 (1989)

13. Lehman, M.M., Belady, L.A. (eds.): Program evolution: processes of software change. Academic Press Professional, Inc., San Diego (1985)

14. Lerner, J., Tirole, J.: Some simple economics of open source. The Journal of Industrial Economics L(2), 197-232 (2002), http: / /www3 . interscience.wiley. com/ cgi-bin/fulltext/118942767/PDFSTART

15. McClure, C.: Software reuse techniques: adding reuse to the system development process. Prentice-Hall, Inc., Upper Saddle River (1997)

16. Morisio, M., Ezran, M., Tully, C.: Success and failure factors in software reuse. IEEE Trans. Softw. Eng. 28(4), 340-357 (2002)

17. Nakakoji, K., Yamamoto, Y., Nishinaka, Y., Kishida, K., Ye, Y.: Evolution patterns of opensource software systems and communities. In: IWPSE 2002: Proceedings of the International Workshop on Principles of Software Evolution, pp. 76-85. ACM, New York (2002), http: //doi.acm.org/10.1145/512035.512055

18. Narduzzo, A., Rossi, A.: The role of modularity in free/open source software development. In: Koch, S. (ed.) Free/Open Source Software Development, pp. 84-102. Idea Group Publishing, Hershey (2004)

19. Parnas, D.L.: On the criteria to be used in decomposing systems into modules, pp. 139-150 (1979)

20. Pressman, R.S.: Software Engineering: A Practitioner's Approach. McGraw-Hill Higher Education, New York (2001)

21. Prieto-Díaz, R.: Implementing faceted classification for software reuse. Commun. ACM 34(5), 88-97 (1991), http: / / doi .acm.org/10.1145/103167.103176

22. Robles, G., Amor, J.J., Gonzalez-Barahona, J.M., Herraiz, I.: Evolution and growth in large libre software projects. In: IWPSE 2005: Proceedings of the Eighth International Workshop on Principles of Software Evolution, pp. 165-174. IEEE Computer Society, Washington (2005)

23. Smith, N., Capiluppi, A., Ramil, J.F.: A study of open source software evolution data using qualitative simulation. Software Process: Improvement and Practice 10(3), 287-300 (2005)

24. Trochim, W.: The Research Methods Knowledge Base, 2nd edn. Atomic Dog Publishing, Cincinnati, http://www.amazon.ca/exec/obidos/redirect?tag= citeulike09-20\&amp; path=ASIN/1931442487

25. Yu, L., Chen, K.: Categorization of common coupling and its application to the maintainability of the linux kernel. IEEE Trans. Softw. Eng. 30(10), 694-706 (2004) 


\section{Appendix}

Table 7. Summary - Evolutionary Models

\begin{tabular}{|c|c|c|c|}
\hline Id & Project & $R^{2}$ & Model \\
\hline $\mathrm{m} 1$ & abiword & 0.97 & $y=1009.771 *$ folder $+325.588 *$ file -210142.563 \\
\hline $\mathrm{m} 2$ & arla & 0.99 & $\mathrm{y}=538.466 *$ folder $+216.124 *$ file -15779.445 \\
\hline $\mathrm{m} 3$ & gaim & 0.97 & $\mathrm{y}=6401.148 *$ folder +8056.888 \\
\hline $\mathrm{m} 4$ & gaim & 0.99 & $\mathrm{y}=423.250 *$ file +664.207 \\
\hline $\mathrm{m} 5$ & gaim & 1 & $\mathrm{y}=38.941 *$ funct +6219.853 \\
\hline $\mathrm{m} 6$ & ganymede & 0.99 & $\mathrm{y}=6013.705 *$ folder $-16.579 *$ file $+18.290 *$ funct -18579.671 \\
\hline $\mathrm{m} 7$ & gdylan & 0.99 & $\mathrm{y}=262.675 *$ file $+12.387 *$ funct +26653.283 \\
\hline $\mathrm{m} 8$ & ghemical & 0.99 & $y=-6665.506 *$ folder $+595.308 *$ file +2807.564 \\
\hline $\mathrm{m} 9$ & gimp_stable & 0.99 & $y=-2249.964 *$ folder $+156.274 *$ file +534680.665 \\
\hline m10 & gimp_stable & 1 & $\mathrm{y}=-380.309 *$ folder $+48.323 *$ funct +66610.831 \\
\hline m11 & gimp_unstable & 1 & $\mathrm{y}=59.860 *$ folder $+53.262 *$ funct -28040.954 \\
\hline m12 & grace & 0.97 & $\mathrm{y}=-5093.335 *$ folder $+622.458 *$ file +40515.292 \\
\hline m13 & htdig & 0.99 & $y=136.588 *$ folder $+54.784 *$ funct +-14221.918 \\
\hline m14 & ksi & 0.93 & $\mathrm{y}=346.067 *$ file $-980.292 *$ folder +36294.173 \\
\hline m15 & htdig & 0.99 & $\mathrm{y}=50.658 *$ file $+48.669 *$ funct -14224.314 \\
\hline m16 & lcrzo & 0.97 & $y=5173.712 *$ folder $+161.054 *$ file $+19.255 *$ funct -12490.114 \\
\hline m17 & motion & 0.95 & $\mathrm{y}=0.000 *$ folder $+77.202 *$ funct -1174.557 \\
\hline m18 & mplayer & 0.99 & $\mathrm{y}=-5057.409 *$ folder $+678.961 *$ file -3626.076 \\
\hline m19 & mrtg & 1 & $\mathrm{y}=81.736 *$ folder $+25.549 *$ funct +2218.992 \\
\hline $\mathrm{m} 20$ & mutt & 0.91 & $\mathrm{y}=-1478.851 *$ folder $+488.015 *$ file +6342.068 \\
\hline $\mathrm{m} 21$ & mutt & 0.97 & $\mathrm{y}=559.707 *$ folder $+71.766 *$ funct +-602.318 \\
\hline m22 & netwib & 0.95 & $\mathrm{y}=3426.807 *$ folder +76321.231 \\
\hline m23 & netwib & 0.99 & $\mathrm{y}=199.451 *$ file +71239.165 \\
\hline $\mathrm{m} 24$ & netwib & 1 & $y=61.597 *$ funct +16614.239 \\
\hline $\mathrm{m} 25$ & rrdtool & 0.95 & $\mathrm{y}=8463.757 *$ folder +3154.039 \\
\hline $\mathrm{m} 26$ & rrdtool & 1 & $\mathrm{y}=1173.715 *$ file +-39939.284 \\
\hline $\mathrm{m} 27$ & rrdtool & 0.99 & $y=230.029 *$ funct -47495.961 \\
\hline $\mathrm{m} 28$ & siagoffice & 0.99 & $\mathrm{y}=1241.303 *$ folder $+311.820 *$ file -15036.930 \\
\hline m29 & vovida & 0.98 & $y=-1244.999 *$ folder $+344.516 *$ file -13420.463 \\
\hline m30 & wine_stable & 0.95 & $\mathrm{y}=4470.605 *$ folder +384237.197 \\
\hline m31 & wine_stable & 0.99 & $\mathrm{y}=777.483 *$ file -254581.635 \\
\hline m32 & wine_stable & 0.99 & $y=36.475 *$ funct +189872.728 \\
\hline m33 & wine_unstable & 0.99 & $\mathrm{y}=5262.688 *$ folder +22096.140 \\
\hline m34 & wine_unstable & 0.99 & $\mathrm{y}=721.482 *$ file -157662.177 \\
\hline $\mathrm{m} 35$ & wine_unstable & 1 & $\mathrm{y}=44.415^{*}$ funct -39778.746 \\
\hline m36 & xfce & 0.98 & $\mathrm{y}=4667.436 *$ folder +8039.892 \\
\hline m37 & xfce & 0.95 & $\mathrm{y}=131.795 *$ funct -73091.477 \\
\hline $\mathrm{m} 38$ & $\mathrm{xmms}$ & 0.94 & $\mathrm{y}=-1525.592 *$ folder $+665.040 *$ file -39494.842 \\
\hline m39 & $\mathrm{xmms}$ & 0.97 & $\mathrm{y}=-560.728 *$ folder $+83.277 *$ funct -27330.425 \\
\hline
\end{tabular}


Table 8. SourceForge sample - modular characteristics (part 1)

\begin{tabular}{|l||cccccc|}
\hline & SLOCs & Fold & File & Funct & Domain Lang \\
\hline perpojo & 1,677 & 10 & 31 & 117 & A & OO \\
\hline moses & 105,955 & 0 & 1,042 & 4,053 & A & OO \\
\hline fn-javabot & 10,142 & 35 & 211 & 279 & A & OO \\
\hline ozone & 63,790 & 141 & 1,018 & 3,920 & B & OO \\
\hline xqilla & 107,320 & 58 & 824 & 2,534 & B & OO \\
\hline fsdb & 241,218 & 362 & 1,715 & 8,506 & B & OO \\
\hline galeon & 93,374 & 11 & 412 & 3,525 & C & P \\
\hline whiteboard & 4,910 & 2 & 13 & 202 & D & php \\
\hline fourever & 15,163 & 28 & 207 & 593 & E & OO \\
\hline hge & 45,654 & 19 & 110 & 800 & F & P \\
\hline zmpp & 15,502 & 24 & 184 & 1,063 & F & OO \\
\hline sudapix & 234 & 8 & 111 & 15,747 & F & P \\
\hline symbolica & 2,623 & 5 & 32 & 67 & F & OO \\
\hline icsDrone & 1,411 & 1 & 14 & 33 & F & P \\
\hline kpictorial & 21 & 3 & 27 & 18,214 & F & sh \\
\hline critical_care & 38,994 & 18 & 185 & 1,051 & F & OO \\
\hline ogce & 350,490 & 1,385 & 3,222 & 13,960 & G & OO \\
\hline cpia & 22,954 & 6 & 25 & 109 & G & P \\
\hline mod_aspdotnet & 2,445 & 4 & 13 & 45 & G & OO \\
\hline xmlnuke & 57,944 & 33 & 395 & 1,623 & G & php \\
\hline wxactivex & 3,264 & 1 & 11 & 37 & G & OO \\
\hline tab-2 & 19,067 & 63 & 334 & 597 & G & php \\
\hline source & 8,786 & 128 & 109 & 162 & G & OO \\
\hline oliver & 1,429 & 2 & 21 & 9 & G & php \\
\hline formproc & 3,514 & 11 & 70 & 134 & G & OO \\
\hline freemind & 28,519 & 30 & 241 & 1,579 & H & OO \\
\hline cdlite & 1,116 & 1 & 6 & 29 & H & OO \\
\hline audiobookcutter & 4,229 & 8 & 37 & 34 & H & OO \\
\hline edict & 2,556 & 1 & 2 & 0 & J & perl \\
\hline qlc & 26,452 & 10 & 203 & 890 & J & OO \\
\hline swtjasperviewer & 3,214 & 4 & 43 & 129 & K & OO \\
\hline QPolymer & 86,971 & 7 & 199 & 652 & L & OO \\
\hline expreval & 3,588 & 2 & 18 & 66 & L & OO \\
\hline eas3pkg & 43,724 & 5 & 101 & 69 & L & f90 \\
\hline neocrypt & 2,135 & 3 & 27 & 21 & M & OO \\
\hline
\end{tabular}


Table 9. SourceForge sample - modular characteristics (part 2)

\begin{tabular}{|l||cccccc|}
\hline \multicolumn{1}{|c||}{} & SLOCs & Fold File & Funct & Domain & Lang \\
\hline \hline juel & 7,284 & 15 & 110 & 404 & $\mathrm{~N}$ & OO \\
\hline csUnit & 16,241 & 41 & 234 & 96 & $\mathrm{~N}$ & cs \\
\hline j_trac & 519 & 34 & 157 & 12,771 & $\mathrm{~N}$ & OO \\
\hline fitnesse & 39,503 & 37 & 631 & 2,321 & $\mathrm{~N}$ & OO \\
\hline ustl & 11,416 & 2 & 94 & 684 & $\mathrm{~N}$ & OO \\
\hline txt2xml & 1,345 & 9 & 25 & 61 & $\mathrm{~N}$ & OO \\
\hline gvision & 101,123 & 9 & 236 & 0 & $\mathrm{~N}$ & pascal \\
\hline seagull & 54,155 & 102 & 362 & 878 & $\mathrm{~N}$ & OO \\
\hline clinkc & 25,846 & 140 & 432 & 919 & $\mathrm{~N}$ & $\mathrm{P}$ \\
\hline simplexml & 1,691 & 3 & 4 & 65 & $\mathrm{~N}$ & $\mathrm{P}$ \\
\hline pf & 213 & 33 & 166 & 84,489 & $\mathrm{O}$ & perl \\
\hline Beobachter & 2,715 & 14 & 49 & 94 & $\mathrm{O}$ & OO \\
\hline blob & 22,056 & 15 & 276 & 496 & $\mathrm{O}$ & $\mathrm{P}$ \\
\hline intermezzo & 34,792 & 15 & 167 & 522 & $\mathrm{O}$ & $\mathrm{P}$ \\
\hline cotvnc & 37,455 & 2 & 225 & 789 & $\mathrm{O}$ & $\mathrm{P}$ \\
\hline
\end{tabular}

Table 10. Debian sample - modular characteristics (part 1)

\begin{tabular}{|l||cccccc|}
\hline \multicolumn{1}{|c||}{} & SLOCs & Fold & File & Funct & Domain Lang \\
\hline \hline kphoneSI & 41,829 & 10 & 263 & 735 & A & OO \\
\hline sylpheed & 106,087 & 6 & 249 & 2,859 & A & P \\
\hline enigmail & 10,790 & 13 & 53 & 86 & A & OO \\
\hline synce-kde & 21,684 & 6 & 95 & 141 & C & sh: \\
\hline txt2html & 3,623 & 2 & 3 & 0 & E & perl: \\
\hline scid & 89,402 & 6 & 151 & 1,179 & F & tcl: \\
\hline netpanzer & 74,368 & 42 & 598 & 2,935 & F & OO/P \\
\hline boson & 224,567 & 78 & 1,272 & 9,246 & F & OO \\
\hline gosa & 107,798 & 101 & 466 & 2,404 & G & php: \\
\hline lirc & 44,753 & 26 & 148 & 785 & G & P \\
\hline openh323 & 234,285 & 30 & 451 & 6,392 & G & OO \\
\hline openafs & 618,553 & 195 & 2,452 & 10,807 & G & P \\
\hline peercast & 22,543 & 8 & 95 & 818 & G & OO \\
\hline slrn & 42,993 & 5 & 91 & 1,189 & G & P \\
\hline cherokee & 54,229 & 17 & 432 & 1,221 & G & P \\
\hline vlc & 401,256 & 129 & 1,378 & 6,250 & H & P \\
\hline cdparanoia & 9,182 & 3 & 37 & 211 & H & P/P \\
\hline kmouth & 5,240 & 3 & 41 & 99 & H & OO \\
\hline rlplot & 69,493 & 1 & 27 & 1,449 & H & OO \\
\hline flac & 56,293 & 42 & 206 & 1,380 & H & P \\
\hline
\end{tabular}


Table 11. Debian sample - modular characteristics (part 2)

\begin{tabular}{|l||cccccc|}
\hline \multicolumn{1}{|c||}{} & SLOCs & Fold & File & Funct & Domain & Lang \\
\hline \hline gwenview & 4,580 & 4 & 62 & 128 & $\mathrm{H}$ & OO \\
\hline prcs1 & 37,360 & 8 & 130 & 663 & $\mathrm{H}$ & OO/P \\
\hline yaml4r & 10,728 & 8 & 31 & 0 & $\mathrm{I}$ & ruby: \\
\hline xmakemol & 18,724 & 1 & 39 & 315 & $\mathrm{I}$ & $\mathrm{P}$ \\
\hline octave_forge & 78,150 & 129 & 409 & 0 & $\mathrm{I}$ & OO/P \\
\hline myphpmoney & 19,434 & 11 & 64 & 153 & $\mathrm{I}$ & php: \\
\hline dia & 146,550 & 43 & 561 & 4,151 & $\mathrm{I}$ & $\mathrm{P}$ \\
\hline grass6 & 107,648 & 115 & 558 & 1,650 & $\mathrm{I}$ & $\mathrm{P}$ \\
\hline geomview & 101,844 & 86 & 771 & 2,748 & $\mathrm{I}$ & $\mathrm{P}$ \\
\hline ProofGeneral & 48,692 & 22 & 134 & 0 & $\mathrm{I}$ & lisp: \\
\hline fte & 51,498 & 2 & 186 & 1,182 & $\mathrm{~K}$ & $\mathrm{OO}$ \\
\hline ruby & 419,942 & 260 & 2,076 & 5,086 & $\mathrm{~K}$ & ruby: \\
\hline EtoileWildMenus & 1,711 & 1 & 21 & 2 & $\mathrm{~K}$ & $\mathrm{OO}$ \\
\hline tcl & 165,306 & 23 & 378 & 2,205 & $\mathrm{~K}$ & $\mathrm{P}$ \\
\hline wxWidgets & $2,142,713$ & 372 & 4,325 & 0 & $\mathrm{~K}$ & $\mathrm{OO}$ \\
\hline libax25 & 11,721 & 1 & 30 & 80 & $\mathrm{~K}$ & sh: \\
\hline liboil & 52,996 & 39 & 304 & 730 & $\mathrm{~K}$ & $\mathrm{P}$ \\
\hline libsoup & 15,012 & 3 & 86 & 494 & $\mathrm{~K}$ & $\mathrm{P}$ \\
\hline Pike & 173,196 & 62 & 408 & 2,302 & $\mathrm{~K}$ & $\mathrm{P}$ \\
\hline shorewall & 25,159 & 6 & 74 & 0 & $\mathrm{~L}$ & sh: \\
\hline acpidump & 2,349 & 1 & 16 & 53 & $\mathrm{~L}$ & $\mathrm{P}$ \\
\hline tiobench & 1,689 & 1 & 8 & 41 & $\mathrm{~L}$ & $\mathrm{P}$ \\
\hline radiusd & 95,967 & 101 & 397 & 1,330 & $\mathrm{~L}$ & $\mathrm{P}$ \\
\hline preludemanager & 10,854 & 15 & 70 & 304 & $\mathrm{~L}$ & $\mathrm{P}$ \\
\hline apmud & 2,502 & 1 & 14 & 45 & $\mathrm{~L}$ & $\mathrm{P}$ \\
\hline clamav & 116,731 & 24 & 339 & 1,056 & $\mathrm{~L}$ & $\mathrm{P}$ \\
\hline tdb & 3,942 & 3 & 19 & 133 & $\mathrm{~L}$ & $\mathrm{P}$ \\
\hline grub & 3,536 & 1 & 7 & 0 & $\mathrm{~L}$ & sh: \\
\hline noteedit & 63,456 & 3 & 139 & 611 & $\mathrm{M}$ & $\mathrm{OO}$ \\
\hline jToolkit & 4,156 & 5 & 32 & 0 & $\mathrm{M}$ & python: \\
\hline
\end{tabular}

